\title{
Memory Effects in Diffusion Like Equation Via Haar Wavelets
}

\author{
I. K. Youssef ${ }^{1}$, A. R. A. Ali ${ }^{2}$ \\ ${ }^{1}$ Department of Mathematics, Ain Shams University, Cairo, Egypt \\ ${ }^{2}$ Department of Mathematics, Baghdad University, Baghdad, Iraq
}

Email address:

Kaoud22@hotmail.com (I. K. Youssef), adil.r.a@ihcoedu.uobaghdad.edu.iq (A. R. A. Ali)

\section{To cite this article:}

I. K. Youssef, A. R. A. Ali. Memory Effects in Diffusion Like Equation Via Haar Wavelets. Pure and Applied Mathematics Journal. Vol. 5, No. 4, 2016, pp. 130-140. doi: 10.11648/j.pamj.20160504.17

Received: July 17, 2016; Accepted: July 26, 2016; Published: August 10, 2016

\begin{abstract}
The memory and hereditary effects of fractional derivatives as well as integral terms are considered in a diffusion like problem. The Haar wavelet operational matrix technique is employed to solve fractional order diffusion equation with time dependent integral term and time dependent boundary condition. The fractional derivative is described in the Caputo sense. The effect of using inverse fractional operator which combines the memory behaviors of the fractional derivatives to all other terms in the equation is disscused. Different Haar bases functions are used $(8,16,32,64)$ and comparison of the wavelet operational matrix is considered. Error analysis is considered. A general numerical example with four subproblems is considered, graphical representation of the different solutions as well as their errors are given.
\end{abstract}

Keywords: Haar Wavelet, Operational Matrix, Fractional Derivative, Fractional Order Diffusion Equation

\section{Introduction}

The problem of solving parabolic partial differential equation in its very simple case

$$
\frac{\partial u}{\partial t}=\frac{\partial^{2} u}{\partial x^{2}}
$$

has been considered by many authors, it is the mathematical model of many physical, engineering, and finance problems. It is well known that the solution of this simple problem requires one initial condition and two boundary conditions. The method of solution of this simple problem depends on the nature of the boundary conditions as well as the initial condition. Moreover, appearance of any other terms in the equation makes the situation more complicated. In this work, we consider a problem of the form

$$
\frac{\partial^{\alpha} u(x, t)}{\partial t^{\alpha}}=\frac{\partial^{2} u(x, t)}{\partial x^{2}}+\sigma \int_{0}^{t} u(x, s) d s+f(x, t)
$$

Where $0<\alpha \leq 1,0 \leq t \leq T$ denotes time, $0 \leq x \leq 1$, and $\sigma=0$ or 1 , with initial condition $u(x, 0)=f(x)$ and boundary conditions $u(0, t)=g_{0}(t), u(1, t)=g_{1}(t)$. The motivation for such equations lies in different branches of physics, in rheology, and especially in the theory of heat conduction when inner heat sources are of special types [1].

Youssef and Shukur, [2] have used the modified variation iteration method to construct an approximate solution.

Equation (2) has many advantages in modeling real problems than equation (1) appearance of fractional time derivatives, non-homogenous terms (in the form of integrals). Except of the fractional derivatives equation (2) is considered in [1] in the form

$$
\frac{\partial u(x, t)}{\partial t}+\mathrm{A} u(x, t)=\int_{0}^{t} K(x, s, u(x, s)) d s+f(x, t)
$$

The number of publications about the fractional calculus has rapidly increased because of some physical processes as anomalous diffusion, complex viscoelasticity, behavior of mechatronic and biological systems, rheology etc. cannot be described adequately by the classical models [3]. The fractional derivatives is understood in the Caputo sense.

Definition (1): The Caputo time fractional derivative of order $\alpha>0$ of the function $u(x, t)$ is defined by $[4,5]$ :

$$
\frac{\partial^{\alpha} u(x, t)}{\partial t^{\alpha}}=\frac{1}{\Gamma(n-\alpha)} \int_{0}^{t}(t-s)^{n-\alpha-1} \frac{d^{n}}{d s^{n}} u(x, s) d s
$$

where $\alpha \in(n-1, n), n \in N$. If $\alpha \in N$, then this will coincide with the classical partial derivative. 
It is generally accepted that differential equations consider the local effects in modeling physical phenomenon, integral equations consider the global behaviors [6], while the fractional derivatives consider the memory and hereditary behaviors [2, 7, 8]. Moreover, the fractional derivatives appears in equation (3) can be translated to the right hand side as

$$
\begin{gathered}
\frac{\partial u(x, t)}{\partial t}=\frac{\partial^{1-\alpha}}{\partial t^{1-\alpha}}\left(\frac{\partial^{2} u(x, t)}{\partial x^{2}}\right) \\
+\sigma \frac{\partial^{1-\alpha}}{\partial t^{1-\alpha}} \int_{0}^{t} u(x, s) d s+\frac{\partial^{1-\alpha}}{\partial t^{1-\alpha}} f(x, t)
\end{gathered}
$$

Thus, the memory and hereditary effects of the fractional derivatives have enforced to the terms on the right hand side and the effects of this well be clear during the numerical calculations.

Due to the developments in computational systems (techniques and devices) and the difficulties in using analytical methods numerical techniques are considered as the master methods for such problems. Among numerical methods the finite differences [2, 4, 7, 8, 9], the weighted residual methods specially their finite element versions also spectral methods or combinations of them are heavily used in solving such problems. Recently, the wavelet methods are extensivelly used espcially the Haar wavelet because its simplecity.

Haar wavelets are made up of pairs of piecewise constant functions and are mathematically the simplest among all the wavelet families. The Haar wavelet is the only real valued wavelet functions which are symmetrical, orthogonal and have a compact support [10]. A good feature of the Haar wavelets is its possibility for integration analytically arbitrary times. The Haar wavelets are very effective for treating singularities, since they can be interpreted as intermediate boundary conditions [11], but the disadvantage of the Haar wavelets is their discontinuity since the derivatives do not exist in the breaking end points.

Chen and Hsiao [12, 13], who first proposed a Haar product matrix and a coeficient matrix, they derived a Haar operational matrix for the integrals of the Haar function vector and put the application for the Haar analysis into the dynamic systems. The technique is to approximate the highest derivative of the differential equation with finite Haar wavelet series. Then integrate this approximation to get the lower order derivatives in the equation. Recently, many authors use this technique to solve the differential or integral equations $[14,15,16,17,18]$.

\section{Haar Wavelets}

Haar wavelet appears in pairs of piecewise constant functions above and below the corresponding axeis in its standard form. Such functions were introduced by Alfred Haar in 1910, Haar showed that certain square wave function could be translated and scaled to create a basis set that span $L_{2}(0,1)$. Years later, it was seen that the system of Haar is a particular wavelet system, and they have been used for solving problems in differential equations only from 1997, [14]. The Haar wavelet family $h_{\mathrm{i}}(x): 0 \leq x \leq 1$ is defined as:

$$
h_{0}(x)= \begin{cases}1, & 0<x \leq 1, \\ 0, & \text { otherwise }\end{cases}
$$

the mother wavelet function $h_{1}(\mathrm{x})$ as:

$$
h_{1}(x)= \begin{cases}1, & 0 \leq x<\frac{1}{2} \\ -1, & \frac{1}{2} \leq x<1 \\ 0, & \text { otherwise }\end{cases}
$$

and For $n \geq 2$ the $n^{\text {th }}$ Haar wavelet functions are defined from $h_{1}(x)$ by translation and dilation operations, i. e

$$
h_{n}(x)=h_{1}\left(2^{j} x-k\right) ; n \geq 1
$$

where $n=2^{j}+k, j \geq 0,0 \leq k<2^{j}$. Haar wavelet functions have orthogonal property in the form

$$
\int_{0}^{1} h_{m}(x) h_{n}(x) d x=2^{-j} \delta_{m n}= \begin{cases}2^{-j}, & m=n=2^{-j}+k, \\ 0, & m \neq n\end{cases}
$$

Accordingly, Haar wavelets are independent functions in the interval $(0,1)$. Wavelet analysis allows representing a function or signal in terms of a set of orthonormal basis functions called wavelets. Haar wavelets are basis for the space $L_{2}[0,1)$, when $j$ increase then the wavelet becomes more narrow, and so $j$ is called the dilatation parameter. The other parameter $k$ effect to wavelet location, for fixed $j$ if $\mathrm{k}$ varying from 0 to $2^{j}-1$ then $h_{n}(x)$ Location varying from $\left[0,2^{-j}\right]$ to $\left[1-2^{-j}, 1\right]$. i.e the interval $[0,1]$ is subdivided into $2^{\mathrm{j}}=m$ subintervals each of length $2^{-\mathrm{j}}$ with center at $\frac{2 k+1}{2 m}$, this is the reason for calling $k$ the translation parameter. It is clear that the wavelet functions have finite support.

\section{Function Approximation}

Using the haar wavelet functions as bases for $L_{2}([0,1])$ enables one to write any function $y(x) \in L_{2}[0,1)$ in the form

$$
y(x)=\sum_{n=0}^{\infty} c_{n} h_{n}(x)
$$

where the coefficients $c_{n}$ are determined by

$$
c_{n}=2^{j} \int_{0}^{1} y(s) h_{n}(s) d s, n \geq 0
$$

with $n=2^{j}+k, j \geq 0$, and $0 \leq k<2^{j}$. Generalally, the series in (10) can be truncated to a finite number of terms. It is interesting to note that if $y(x)$ is piecewise constant, or may be approximated as piecewise constant during each subinterval, then $y(x)$ will be terminated at finite number of terms

$$
y(x)=\sum_{n=0}^{m-1} c_{n} h_{n}(x)=\boldsymbol{C}_{(m)}^{T} \boldsymbol{h}_{(m)}(x)
$$

Where the coefficient vector $\boldsymbol{C}_{(m)}^{T}$ and the Haar function vector $\boldsymbol{h}_{(m)}(x)$ are define as

$$
\boldsymbol{C}_{(m)}^{T}=\left[c_{0}, c_{1}, \cdots, c_{m-1}\right]
$$




$$
\boldsymbol{h}_{(m)}(x)=\left[h_{0}(x), h_{1}(x), h_{m-1}(x)\right]^{T}
$$

where $T$ means transpose and $m=2^{j}$.

The first four Haar function vectors which $x=\left[\frac{1}{8}, \frac{3}{8}, \frac{5}{8}, \frac{7}{8}\right]$ can be expressed the following [12]

$$
\begin{gathered}
\boldsymbol{h}_{(4)}(1 / 8)=[1,1,1,0]^{T}, \boldsymbol{h}_{(4)}(3 / 8)=[1,1,-1,0]^{T}, \\
\boldsymbol{h}_{(4)}(5 / 8)=[1,-1,0,1]^{T}, \boldsymbol{h}_{(4)}(7 / 8)=[1,-1,0,1]^{T}
\end{gathered}
$$

this can be written in matrix form as

$$
\begin{aligned}
\boldsymbol{H}_{(4)}=\left[\boldsymbol{h}_{(4)}(1 / 8)\right. & \left., \boldsymbol{h}_{(4)}(3 / 8), \boldsymbol{h}_{(4)}(5 / 8), \boldsymbol{h}_{(4)}(7 / 8)\right] \\
= & {\left[\begin{array}{llll}
1 & 1 & 1 & 1 \\
1 & 1 & -1 & -1 \\
1 & -1 & 0 & 0 \\
0 & 0 & 1 & -1
\end{array}\right] }
\end{aligned}
$$

In general, we have

$$
\boldsymbol{H}_{(m)}=\left[\boldsymbol{h}_{(m)}\left(\frac{1}{2 m}\right), \boldsymbol{h}_{(m)}\left(\frac{3}{2 m}\right), \cdots, \boldsymbol{h}_{(m)}\left(\frac{2 m-1}{2 m}\right)\right]
$$

where $\boldsymbol{H}_{(1)}=[1], \boldsymbol{H}_{(2)}=\left[\begin{array}{ll}1 & 1 \\ 1 & -1\end{array}\right]$. The integration of the vector $\boldsymbol{h}_{(m)}(x)$ is given by

$$
\int_{0}^{\mathrm{x}} \boldsymbol{h}_{(m)}(s) d s=\boldsymbol{P}_{(m)} \boldsymbol{h}_{(m)}(x)
$$

Where $0 \leq x<1$ and $\boldsymbol{P}_{(m)}$ is the $m \times m$ operational matrix. Chen and Hsiao [12], who first proposed a Haar product matrix and a coefficient matrix, proved that

$$
\boldsymbol{P}_{(m)}=\frac{1}{2 m}\left[\begin{array}{ll}
2 m \boldsymbol{P}_{(m / 2)} & -\boldsymbol{H}_{(m / 2)} \\
\boldsymbol{H}_{(m / 2)}^{-1} & O
\end{array}\right]
$$

Where $\boldsymbol{P}_{(1)}=\left[\frac{1}{2}\right], \boldsymbol{P}_{(2)}=\frac{1}{4}\left[\begin{array}{ll}2 & -1 \\ 1 & 0\end{array}\right]$,

$$
\boldsymbol{P}_{(4)}=\frac{1}{16}\left[\begin{array}{cccc}
8 & -4 & -2 & -2 \\
4 & 0 & -2 & 2 \\
1 & 1 & 0 & 0 \\
1 & -1 & 0 & 0
\end{array}\right] \text { and so on. }
$$

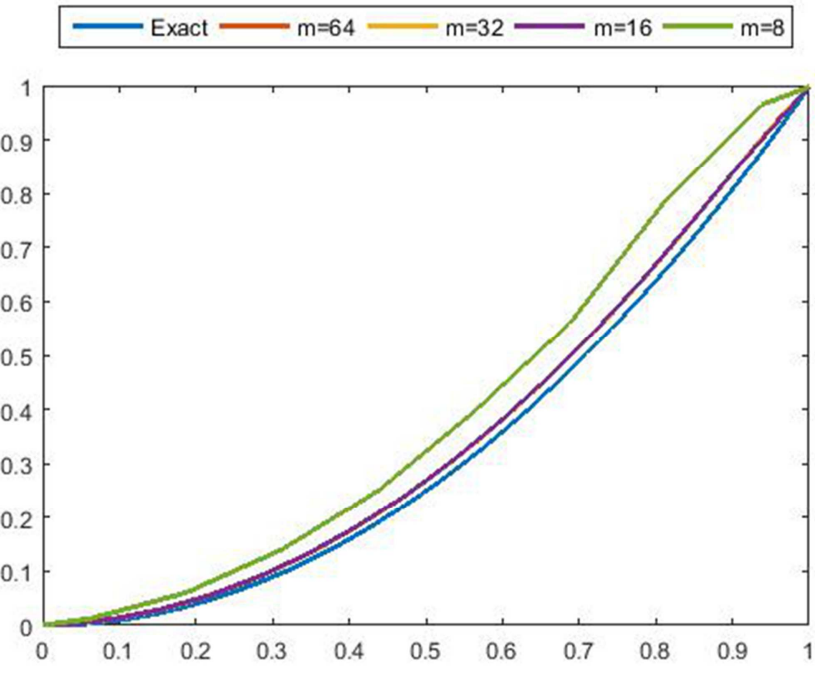

(1 a): Numerical Solutions

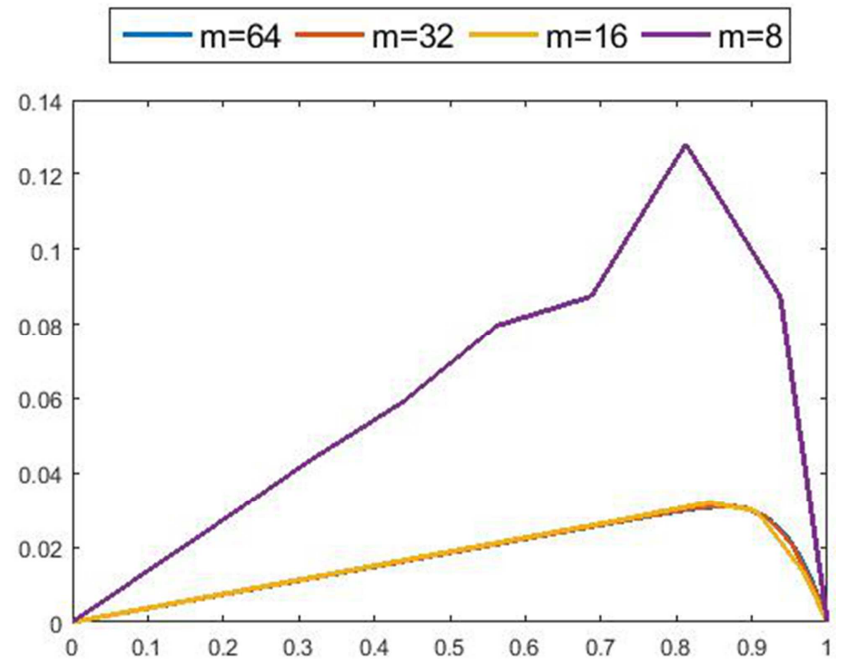

(1 b): Maximum errors $\mathrm{M}_{\Delta}$

Figure 1. Comparison between the exact solution and the approximate solutions of the partial differential equation for Case 1 equation (39).
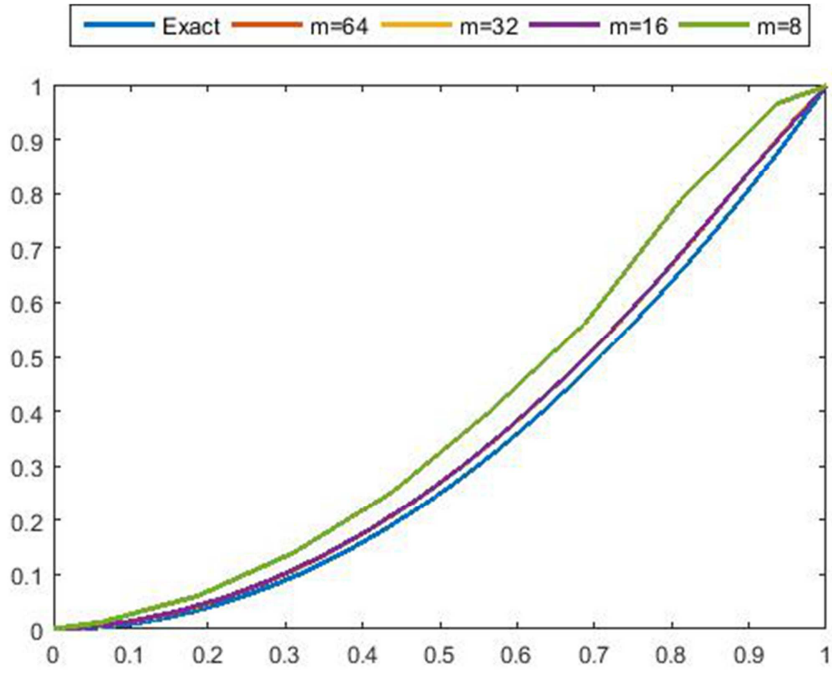

(2 a): Numerical Solutions

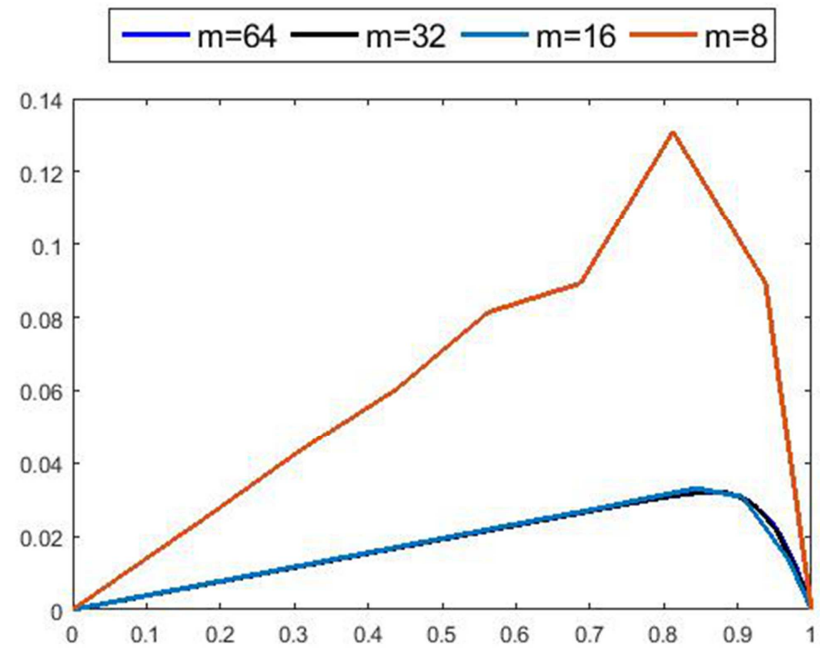

(2 b): Maximum errors $\mathrm{M}_{\Delta}$

Figure 2. Comparison between the exact solution and the approximate solutions of the partial differential equation for Case 2 equation (42). 


\section{Method of Solution}

The basic idea is to write the dominant derivative term appear in the problem as a finite series in the form

$$
\dot{u}^{\prime \prime}(x, t)=\sum_{n=0}^{m-1} c_{n}\left(t_{s}\right) h_{n}(x)=\boldsymbol{C}_{(m)}^{T} \boldsymbol{h}_{(m)}(x)
$$

The dominant derivative term means the term which contains all the highest derivatives in only one term, the dot is used to denote derivatives with respect time and the primes means differentiation with respect to the space $x$.

$$
\begin{gathered}
u^{\prime \prime}(x, t)=u^{\prime \prime}\left(x, t_{s}\right)+\left(t-t_{s}\right) \boldsymbol{C}_{(m)}^{T} \boldsymbol{h}_{(m)}(x) \\
u^{\prime}(x, t)=u^{\prime}\left(x, t_{s}\right)+u^{\prime}(0, t)-u^{\prime}\left(0, t_{s}\right)+\left(t-t_{s}\right) \boldsymbol{C}_{(m)}^{T} \boldsymbol{P}_{(m)} \boldsymbol{h}_{(m)}(x) \\
u(x, t)=\left(t-t_{s}\right) \boldsymbol{C}_{(m)}^{T} \boldsymbol{P}_{(m)}^{2} \boldsymbol{h}_{(m)}(x)+u\left(x, t_{s}\right)-u\left(0, t_{s}\right)+u(0, t)+x\left[u^{\prime}(0, t)-u^{\prime}\left(0, t_{s}\right)\right] \\
\dot{u}(x, t)=\boldsymbol{C}_{(m)}^{T} \boldsymbol{P}_{(m)}^{2} \boldsymbol{h}_{(m)}(x)+\dot{u}(0, t)+x \dot{u}^{\prime}(0, t)
\end{gathered}
$$

From the boundary conditions, we can get $u\left(0, t_{s}\right)=g_{0}\left(t_{s}\right), u\left(1, t_{s}\right)=g_{1}\left(t_{s}\right), \dot{u}(0, t)=g_{0}(t), \dot{u}(1, t)=g_{1}(t)$, Putting $x=1$ in formula (22) and (23) to get

$$
\begin{gathered}
u^{\prime}(0, t)=u^{\prime}\left(0, t_{s}\right)-\left(t-t_{s}\right) \boldsymbol{C}_{(m)}^{T} \boldsymbol{P}_{(m)}^{2} \boldsymbol{h}_{(m)}(x)+g_{1}(t)-g_{1}\left(t_{s}\right)+g_{0}\left(t_{s}\right)-g_{0}(t) \\
\dot{u}^{\prime}(0, t)=-\boldsymbol{C}_{(m)}^{T} \boldsymbol{P}_{(m)}^{2} \boldsymbol{f}+g_{1}(t)-g_{0}(t)
\end{gathered}
$$

where the vector $f$ is defined as $f=[1,0, \cdots, 0]^{T}$. Substituting formula (24) and (25) into equations (20) to (23) and rewrite the results by assuming $x=x_{l}, t=t_{s+1}$ and $d t=\left(t-t_{s}\right)$ to obtain

$$
\begin{gathered}
u^{\prime \prime}\left(x_{l}, t_{s+1}\right)=u^{\prime \prime}\left(x_{l}, t_{s}\right)+d t \boldsymbol{C}_{(m)}^{T} \boldsymbol{h}_{(m)}(x) \\
u^{\prime}\left(x_{l}, t_{s+1}\right)=u^{\prime}\left(x_{l}, t_{s}\right)+d t \boldsymbol{C}_{(m)}^{T} \boldsymbol{P}_{(m)} \boldsymbol{h}_{(m)}(x)-d t \boldsymbol{C}_{(m)}^{T} \boldsymbol{P}_{(m)} \boldsymbol{f}+g_{1}\left(t_{s+1}\right)-g_{1}\left(t_{s}\right)+g_{0}\left(t_{s}\right)-g_{0}\left(t_{s+1}\right)
\end{gathered}
$$

$u\left(x_{l}, t_{s+1}\right)=d t \boldsymbol{C}_{(m)}^{T} \boldsymbol{P}_{(m)}^{2} \boldsymbol{h}_{(m)}\left(x_{l}\right)+u\left(x_{l}, t_{s}\right)-g_{0}\left(t_{s}\right)+g_{0}\left(t_{s+1}\right)+x_{l}\left[-d t \boldsymbol{C}_{(m)}^{T} \boldsymbol{P}_{(m)} \boldsymbol{f}+g_{1}\left(t_{s+1}\right)-g_{1}\left(t_{s}\right)+g_{0}\left(t_{s}\right)-g_{0}\left(t_{s+1}\right)\right]$

$$
\dot{u}\left(x_{l}, t_{s+1}\right)=\boldsymbol{C}_{(m)}^{T} \boldsymbol{P}_{(m)}^{2} \boldsymbol{h}_{(m)}\left(x_{l}\right)+\dot{u}\left(0, t_{s+1}\right)+x_{l}\left[-\boldsymbol{C}_{(m)}^{T} \boldsymbol{P}_{(m)}^{2} \boldsymbol{f}+g_{\mathrm{i}}\left(t_{s+1}\right)-g_{0}\left(t_{s+1}\right)\right]
$$

And for the integral term

$$
\begin{aligned}
\int_{0}^{t} u(x, s) d s= & \left(\frac{t_{s+1}^{2}}{2}-t_{s+1} t_{s}\right) \boldsymbol{C}_{(m)}^{T} \boldsymbol{P}_{(m)}^{2} \boldsymbol{h}_{(m)}\left(x_{l}\right)+t_{s+1} u\left(x_{l}, t_{s}\right)-x_{l}\left(\frac{t_{s+1}^{2}}{2}-t_{s+1} t_{s}\right) \boldsymbol{C}_{(m)}^{T} \boldsymbol{P}_{(m)} \boldsymbol{f}+t_{s+1}\left[x_{l}\left(g_{0}\left(t_{s}\right)-g_{1}\left(t_{s}\right)\right)-\right. \\
& \left.g_{0}\left(t_{s}\right)\right]+\int_{0}^{t}\left[g_{0}(s)+x_{l}\left(g_{1}(s)-g_{0}(s)\right)\right] d s
\end{aligned}
$$

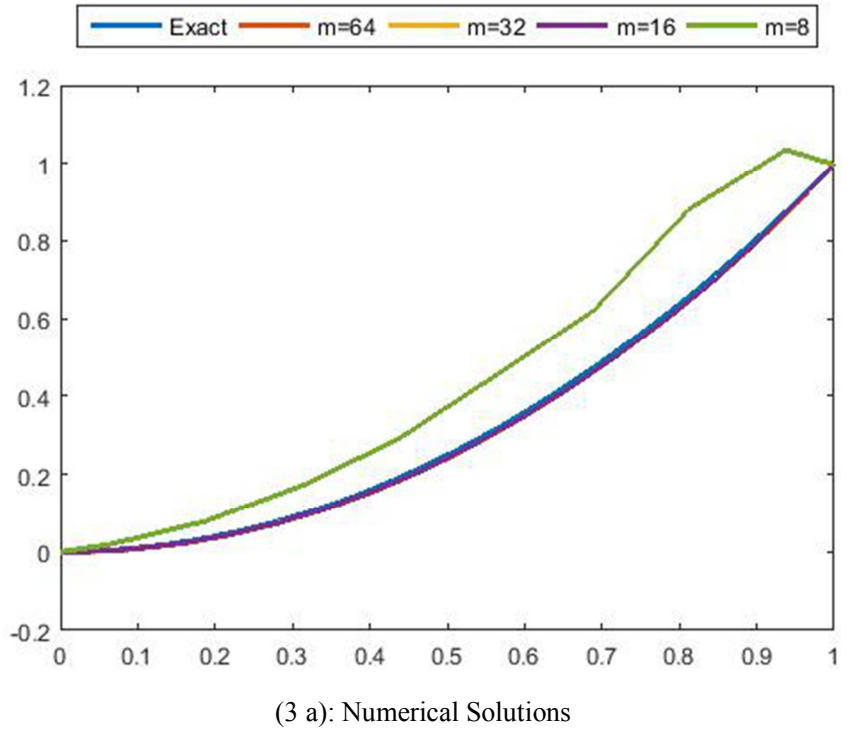




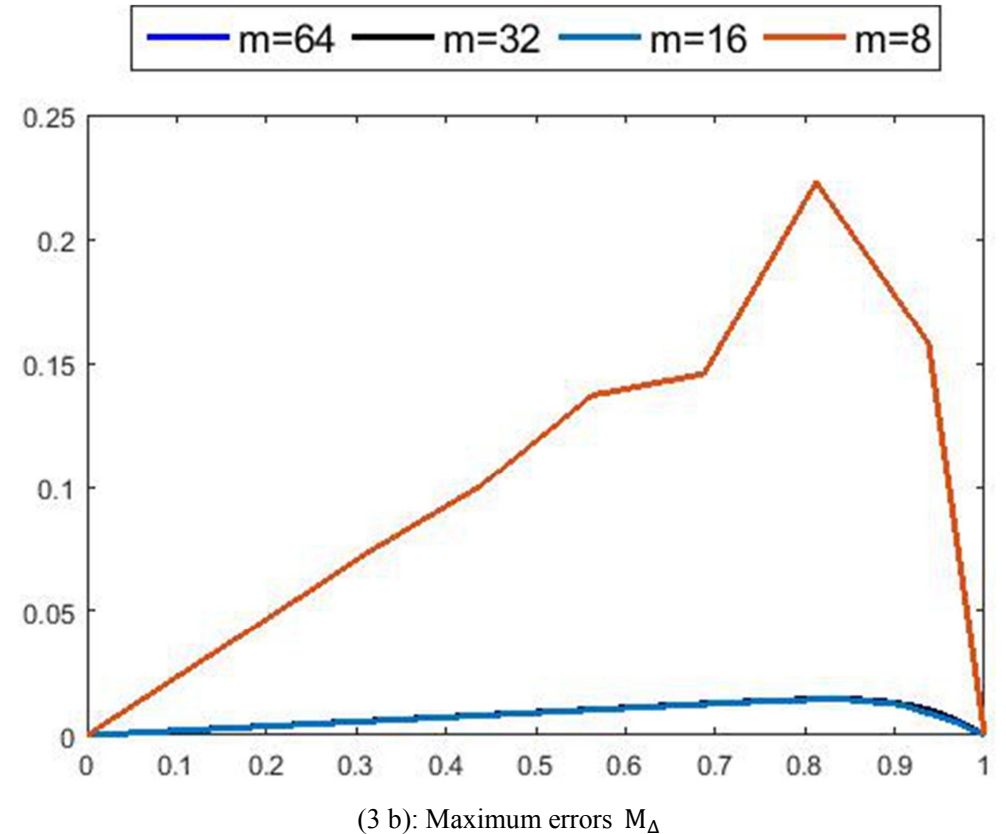

Figure 3. Comparison between the exact solution and the approximate solutions of the partial differential equation for Case 3 equation (44) with $\alpha=0.9$.

The fractional derivative $\frac{\partial^{\alpha} u(x, t)}{\partial t^{\alpha}}$ is understood in the Caputo sense defined above. Accordinglly, the fractional derivative $\frac{\partial^{\alpha} u(x, t)}{\partial t^{\alpha}}$ can be rewritten as:

$$
\frac{\partial^{\alpha} u(x, t)}{\partial t^{\alpha}}=\frac{1}{\Gamma(1-\alpha)} \int_{0}^{t}(t-s)^{-\alpha}\left[\boldsymbol{C}_{(m)}^{T} \boldsymbol{P}_{(m)}^{2} \boldsymbol{h}_{(m)}(x)+g_{0}^{\prime}\left(t_{s+1}\right)+x_{l}\left[-\boldsymbol{C}_{(m)}^{T} \boldsymbol{P}_{(m)}^{2} \boldsymbol{f}+g_{1}^{\prime}\left(t_{s+1}\right)-g_{0}^{\prime}\left(t_{s+1}\right)\right]\right] d s
$$

which can be rearranged as

$$
\frac{\partial^{\alpha} u(x, t)}{\partial t^{\alpha}}=\frac{1}{\Gamma(1-\alpha)} \int_{0}^{t}(t-s)^{-\alpha}\left[x_{l}\left[g_{i}\left(t_{s+1}\right)-g_{0}\left(t_{s+1}\right)\right]+g_{0}\left(t_{s+1}\right)\right] d s+\frac{1}{\Gamma(1-\alpha)}\left[\boldsymbol{C}_{(m)}^{T} \boldsymbol{P}_{(m)}^{2} \boldsymbol{h}_{(m)}(x)-x_{l} \boldsymbol{C}_{(m)}^{T} \boldsymbol{P}_{(m)}^{2} \boldsymbol{f}\right]\left(\frac{t^{1-\alpha}}{1-\alpha}\right)
$$

and $\frac{\partial^{1-\alpha}}{\partial t^{1-\alpha}}\left[\frac{\partial^{2} u(x, t)}{\partial x^{2}}\right]$ can be calculated as

$$
\begin{aligned}
\frac{\partial^{1-\alpha}}{\partial t^{1-\alpha}}\left[\frac{\partial^{2} u(x, t)}{\partial x^{2}}\right] & =\frac{\partial^{\alpha-1} u(x, t)}{\partial t^{\alpha-1}}\left[u^{\prime \prime}\left(x, t_{s}\right)+\left(t-t_{s}\right) \boldsymbol{C}_{(m)}^{T} \boldsymbol{h}_{(m)}(x)\right] \\
& =\frac{1}{\Gamma(1-(1-\alpha))} \int_{0}^{t}(t-s)^{-(1-\alpha)} \frac{d}{d s}\left[u^{\prime \prime}\left(x, t_{s}\right)+\left(s-t_{s}\right) \boldsymbol{C}_{(m)}^{T} \boldsymbol{h}_{(m)}(x)\right] d s \\
& =\frac{1}{\Gamma(\alpha)} \int_{0}^{t}(t-s)^{-(1-\alpha)}\left[\boldsymbol{C}_{(m)}^{T} \boldsymbol{h}_{(m)}(x)\right] d s=\frac{-t}{\alpha \Gamma(\alpha)} \boldsymbol{C}_{(m)}^{T} \boldsymbol{h}_{(m)}(x)
\end{aligned}
$$

then by equation (30) we can get

$$
\begin{gathered}
\frac{\partial^{1-\alpha}}{\partial t^{1-\alpha}} \int_{0}^{t} u(x, s) d s=\left(\frac{\Gamma(3)}{2 \Gamma(2+\alpha)} t_{s+1}^{1+\alpha}-\frac{\Gamma(2)}{\Gamma(1+\alpha)} t_{s+1}^{\alpha} t_{s}\right) \boldsymbol{C}_{(m)}^{T} \boldsymbol{P}_{(m)}^{2} \boldsymbol{h}_{(m)}\left(x_{l}\right)+\frac{\Gamma(2)}{\Gamma(1+\alpha)} t_{s+1}^{\alpha} u\left(x_{l}, t_{s}\right)+\frac{\Gamma(2)}{\Gamma(1+\alpha)} t_{s+1}^{\alpha}\left[x _ { l } \left(g_{0}\left(t_{s}\right)-\right.\right. \\
\left.\left.g_{1}\left(t_{s}\right)\right)-g_{0}\left(t_{s}\right)\right]+\frac{\partial^{1-\alpha}}{\partial t^{1-\alpha}} \int_{0}^{t}\left[g_{0}(s)+x_{l}\left(g_{1}(s)-g_{0}(s)\right)\right] d s-x_{l}\left(\frac{\Gamma(3)}{2 \Gamma(2+\alpha)} t_{s+1}^{1+\alpha}-\frac{\Gamma(2)}{\Gamma(1+\alpha)} t_{s+1}^{\alpha} t_{s}\right) \boldsymbol{C}_{(m)}^{T} \boldsymbol{P}_{(m)} \boldsymbol{f}
\end{gathered}
$$

Substitution equations (26), (29), (30), (32), (33) and (34) in equation (2) or (5), we find

$$
\begin{gathered}
\frac{\partial^{\alpha} u(x, t)}{\partial t^{\alpha}}=u^{\prime \prime}\left(x_{l}, t_{s+1}\right)+\sigma \int_{0}^{t} u(x, s) d s+f\left(x_{l}, t_{s+1}\right) \\
\frac{\partial u(x, t)}{\partial \mathrm{t}}=\frac{\partial^{1-\alpha}}{\partial t^{1-\alpha}} u^{\prime \prime}\left(x_{l}, t_{s+1}\right)+\sigma \frac{\partial^{1-\alpha}}{\partial t^{1-\alpha}} \int_{0}^{t} u(x, s) d s+\frac{\partial^{1-\alpha}}{\partial t^{1-\alpha}} f\left(x_{l}, t_{s+1}\right)
\end{gathered}
$$

The Haar coefficients vector $\boldsymbol{C}_{(m)}$ are calculated from the system of linear equations results from (35) or (36). The

solution is found according to equation (28). 


\section{Error Estimates}

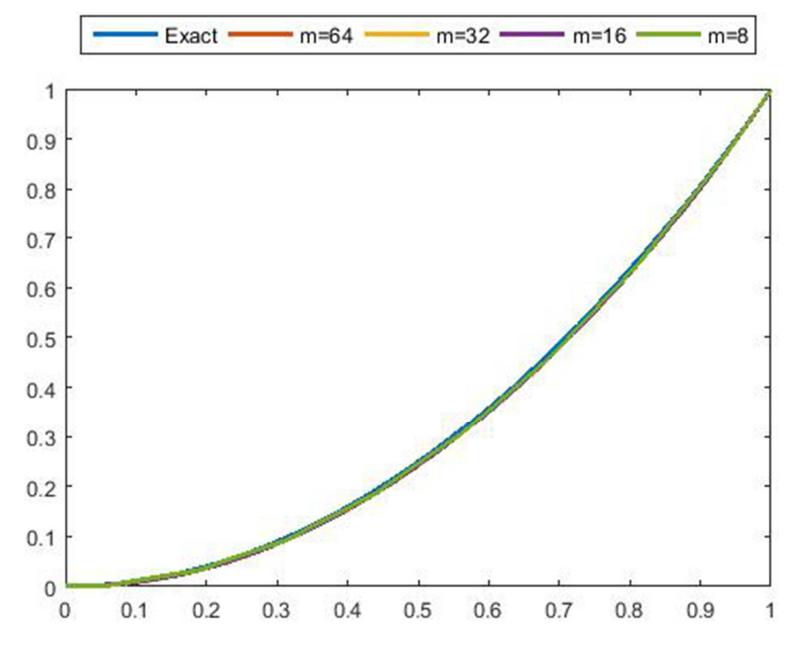

(4 a): Numerical Solutions

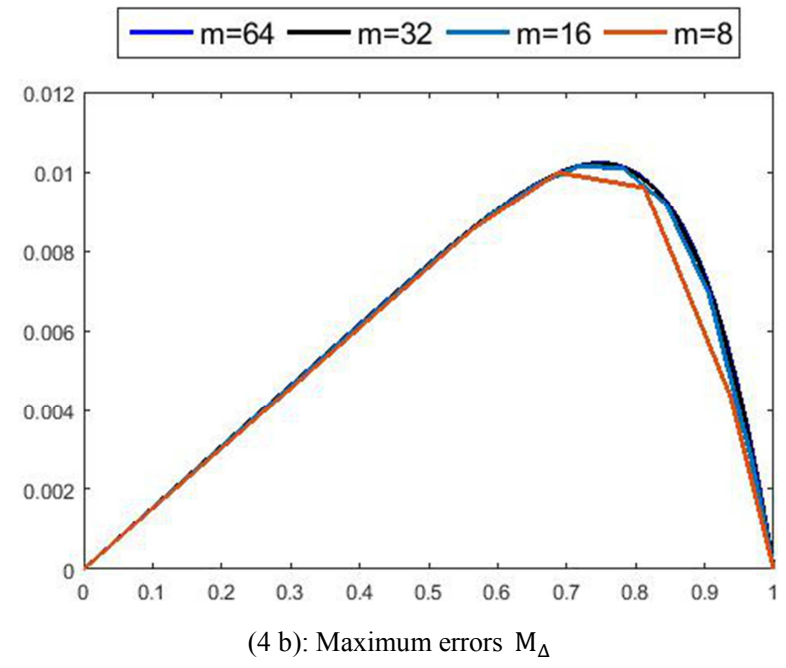

Figure 4. Comparison between the exact solution and the approximate solutions of the partial differential equation for Case 3 equation (44) with $\alpha=0.7$.

In order to estimate the accuracy of the obtained solution define the average error $\sigma_{\Delta}$ as global error estimate and Maximum error $M_{\Delta}$ as local error estimate as follows

$$
\begin{gathered}
\Delta(l)=u_{e x}\left(\frac{2 l-1}{2 m}, T\right)-u\left(\frac{2 l-1}{2 m}, T\right), l=1,2,3, \cdots, m \\
M_{\Delta}=\operatorname{Max}|\Delta(l)| \\
\sigma_{\Delta}=\frac{\sum_{l=1}^{l=m}|\Delta(l)|}{m}
\end{gathered}
$$

It is interesting to note that increasing the number of collocation points not always give better approximate solution, in some cases increasing the number of collocation points may turn the coefficient matrix of the resultant algebraic system to be nearly singular, [14].

\section{Numerical Example}

Equation (2) descripes a general problem and its solution requires an initial condition $u(x, 0)=x^{2}$ and two boundary conditions $u(0, t)=0$ and $u(1, t)=e^{-t}$. In order to recognize the effect of terms we divided the problem to four cases and the force term $f(x, t)$ is used to adobt the exact solution to be $u(x, t)=x^{2} e^{-t}$. All results are given with $\Delta t=0.00025$, with ten time steps and $T=0.0025$.

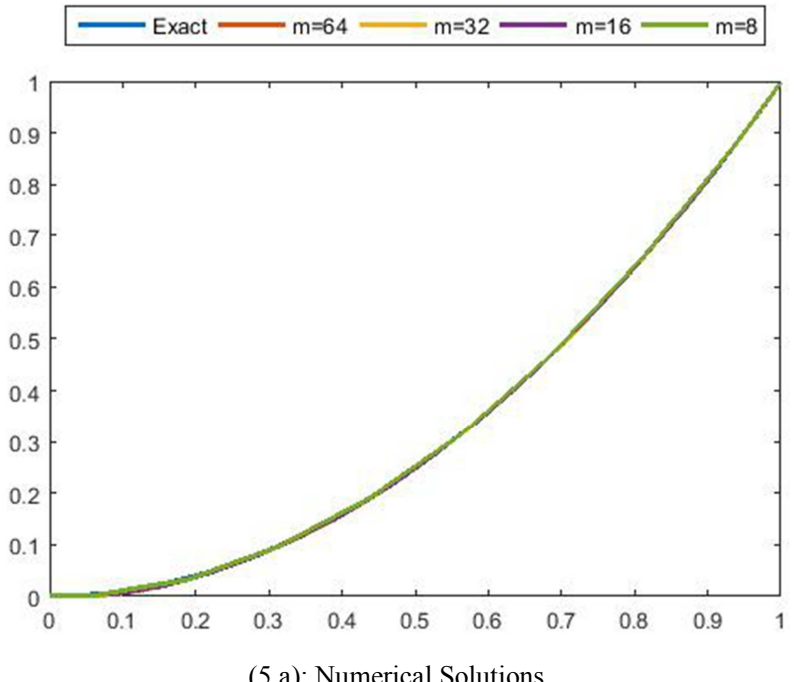

(5 a): Numerical Solutions

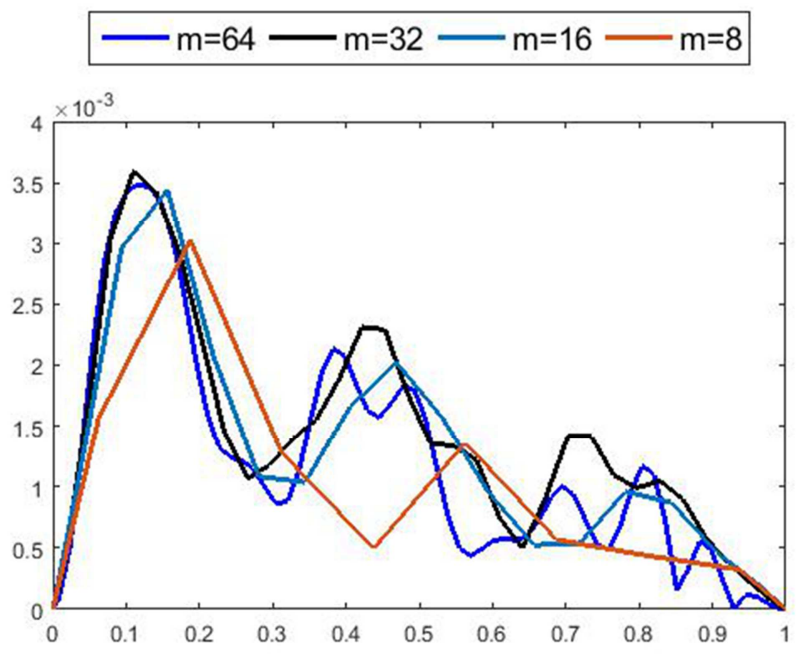

(5 b): Maximum errors $\mathrm{M}_{\Delta}$

Figure 5. Comparison between the exact solution and the approximate solutions of the partial differential equation for Case 3 equation (47) with $\alpha=0.9$.

\subsection{Case 1}

The classical (integer) diffusion equation with non homogenous term and without integral term $\sigma=0, \alpha=1$, then equation (2) take the form

$$
\frac{\partial u(x, t)}{\partial t}=\frac{\partial^{2} u(x, t)}{\partial x^{2}}-\left(x^{2}+2\right) e^{-t}
$$

Then equation (29) will be

$\dot{u}\left(x_{l}, t_{s}\right)=\boldsymbol{C}_{(m)}^{T} \boldsymbol{P}_{(m)}^{2} \boldsymbol{h}_{(m)}(x)-x_{l}\left[\boldsymbol{C}_{(m)}^{T} \boldsymbol{P}_{(m)}^{2} \boldsymbol{f}+e^{-t}\right]$

Substitute equations (40) and (26) in equation (39) we 
obtain

$$
-d t \boldsymbol{C}_{(m)}^{T} \boldsymbol{h}_{(m)}(x)-u^{\prime \prime}\left(x_{l}, t_{s}\right)+e^{-t_{s+1}}\left(x_{l}^{2}+2\right)=0
$$

$$
\boldsymbol{C}_{(m)}^{T} \boldsymbol{P}_{(m)}^{2} \boldsymbol{h}_{(m)}(x)-x_{l}\left[\boldsymbol{C}_{(m)}^{T} \boldsymbol{P}_{(m)}^{2} \boldsymbol{f}+e^{-t_{s+1}}\right]
$$

In this case equation (5) take the same form as equation (2)

Exact $-\mathrm{m}=64 \longrightarrow \mathrm{m}=32 \longrightarrow \mathrm{m}=8$

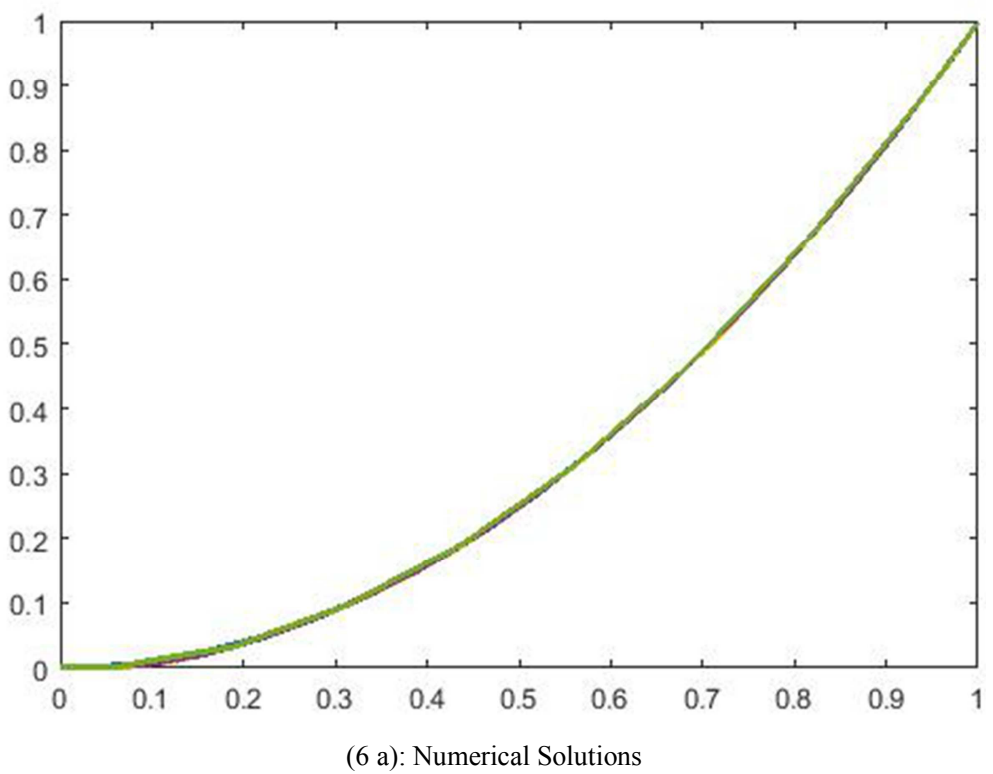

\section{$m \mathrm{~m}=64-\mathrm{m}=32-\mathrm{m}=16-8$}

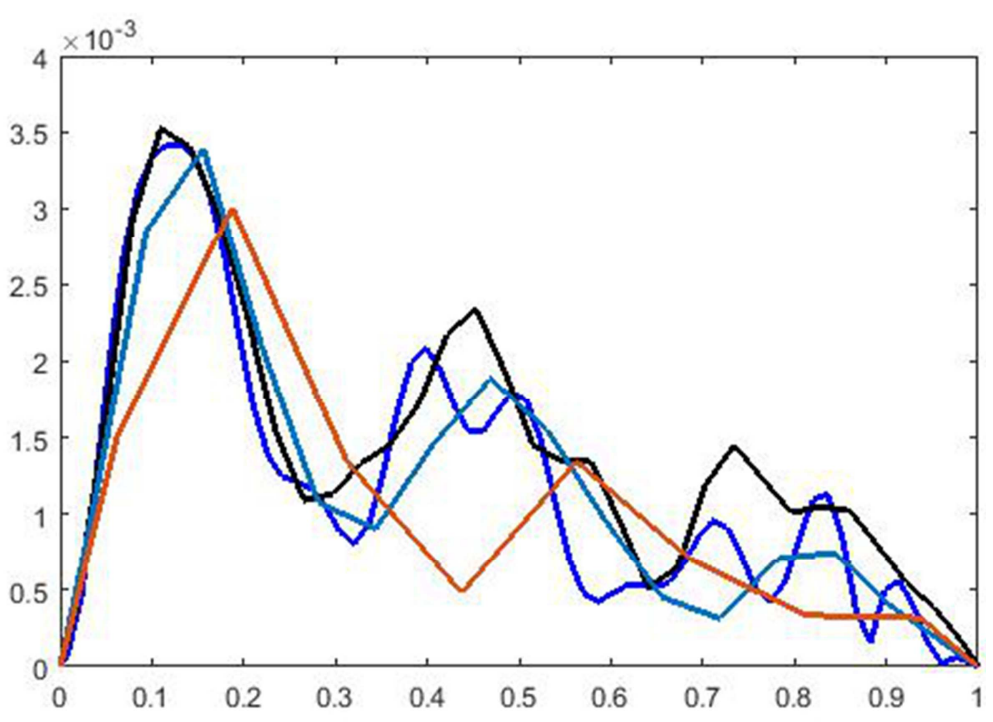

(6 b): Maximum errors $M_{\Delta}$

Figure 6. Comparison between the exact solution and the approximate solutions of the partial differential equation for Case 3 equation (47) with $\alpha=0.7$.

\subsection{Case 2}

The classical (integer) diffusion equation with time dependent integral term and non homogenous term, $\sigma=1$, $\alpha=1$ then equation (1) take the form

$$
\frac{\partial u(x, t)}{\partial t}=\frac{\partial^{2} u(x, t)}{\partial x^{2}}+\int_{0}^{t} u(x, s) d s-x^{2}-2 e^{-t}
$$

Calculate equation (30) and Substitute it with equations (40) and (26) in equation (42) to get

$$
\begin{array}{r}
C_{(m)}^{T} P_{(m)}^{2} h_{(m)}(x)-x_{l}\left[C_{(m)}^{T} P_{(m)}^{2} f+e^{-t_{s+1}}\right]-d t C_{(m)}^{T} h_{(m)}(x)-u^{\prime \prime}\left(x_{l}, t_{s}\right)+x^{2}+2 e^{-t_{s+1}}- \\
\left(\frac{t_{s+1}^{2}}{2}-t_{s+1} t_{s}\right) C_{(m)}^{T} P_{(m)}^{2} h_{(m)}(x)-t_{s+1} u\left(x_{l}, t_{s}\right)+x_{l}\left(\frac{t_{s+1}^{2}}{2}-t_{s+1} t_{s}\right) C_{(m)}^{T} P_{(m)} f-t_{s+1} x_{l} e^{-t_{s+1}}-x_{l}\left(1-e^{-t_{s+1}}\right)=0
\end{array}
$$


In this case equation (5) take the same form as equation (2)
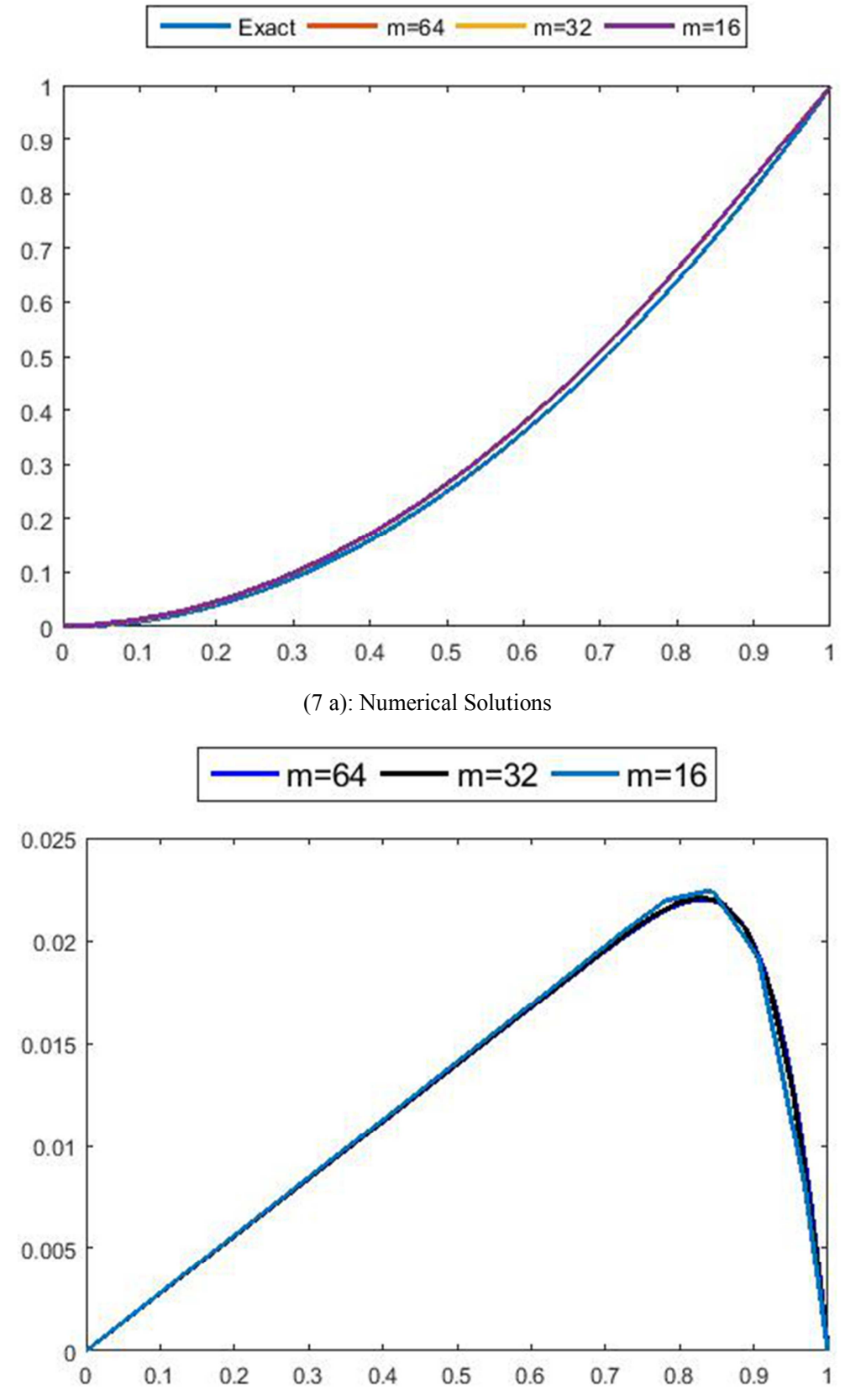

(7 b): Maximum errors $M_{\Delta}$

Figure 7. Comparison between the exact solution and the approximate solutions of the partial differential equation for Case 4 equation (49) with $\alpha=0.9$.

\subsection{Case 3}

The fractional time diffusion equation, when $\sigma=0$, $0<\alpha \leq 1$ then equation (2) take the form

$$
\frac{\partial^{\alpha} u(x, t)}{\partial t^{\alpha}}=\frac{\partial^{2} u(x, t)}{\partial x^{2}}+x^{2} \frac{\partial^{\alpha}}{\partial t^{\alpha}} e^{-t}-2 e^{-t}
$$

$$
\frac{1}{\Gamma(1-\alpha)}\left(\boldsymbol{C}_{(m)}^{T} \boldsymbol{P}_{(m)}^{2} \boldsymbol{h}_{(m)}\left(x_{l}\right)-x_{l} \boldsymbol{C}_{(m)}^{T} \boldsymbol{P}_{(m)}^{2} \boldsymbol{f}\right)\left(\frac{t_{s+1}^{1-\alpha}}{1-\alpha}\right)+x_{l} \frac{\partial^{\alpha}}{\partial t^{\alpha}} e^{-t_{s+1}}-d t \boldsymbol{C}_{(m)}^{T} \boldsymbol{h}_{(m)}\left(x_{l}\right)-u^{\prime \prime}\left(x_{l}, t_{s}\right)-x^{2} \frac{\partial^{\alpha}}{\partial t^{\alpha}} e^{-t_{s+1}}+2 e^{-t_{s+1}}=0
$$

and equation (5) take the form

Substitute equations (45) and (26) in equation (44) we gain

$$
\frac{\partial u(x, t)}{\partial t}=\frac{\partial^{1-\alpha}}{\partial t^{1-\alpha}}\left(\frac{\partial^{2} u(x, t)}{\partial x^{2}}\right)-x^{2} e^{-t}-2 \frac{\partial^{1-\alpha}}{\partial t^{1-\alpha}} e^{-t}
$$


Substitute equations (40) and (33) in equation (47), we gain $\boldsymbol{C}_{(m)}^{T} \boldsymbol{P}_{(m)}^{2} \boldsymbol{h}_{(m)}\left(x_{l}\right)-x_{l}\left[\boldsymbol{C}_{(m)}^{T} \boldsymbol{P}_{(m)}^{2} \boldsymbol{f}+e^{-t_{s+1}}\right]+x_{l}^{2} e^{-t_{s+1}}+$ $\frac{t}{\alpha \Gamma(\alpha)} \boldsymbol{C}_{(m)}^{T} \boldsymbol{h}_{(m)}\left(x_{l}\right)+2 \frac{\partial^{1-\alpha}}{\partial t^{1-\alpha}} e^{-t^{S+1}}=0$
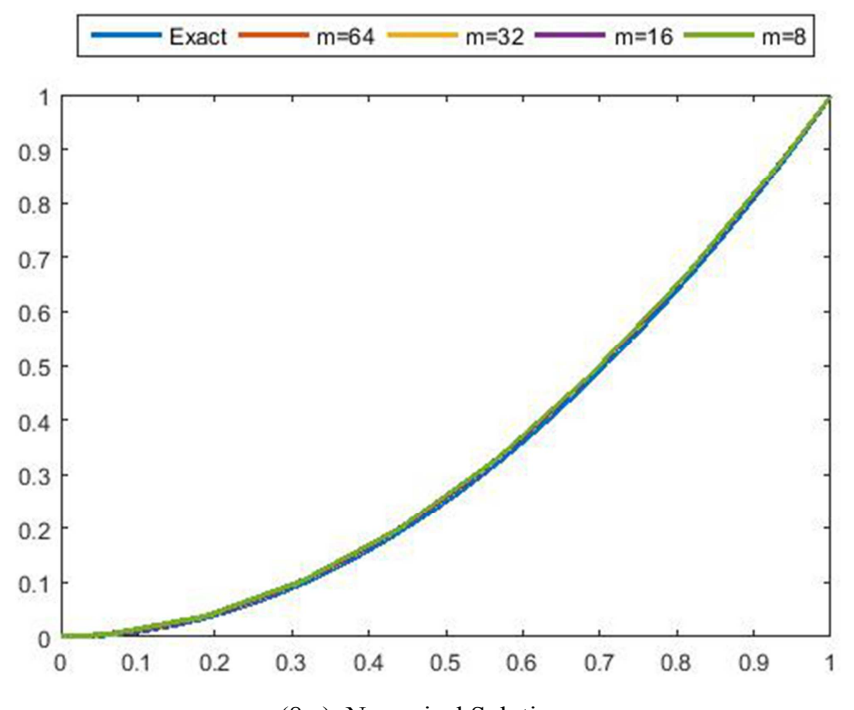

(8 a): Numerical Solutions

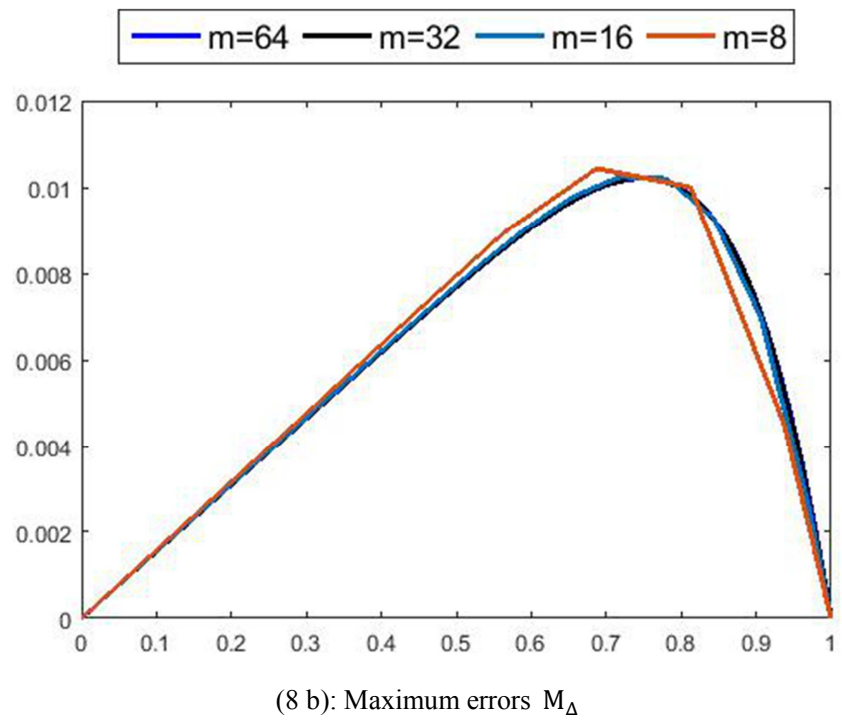

Figure 8. Comparison between the exact solution and the approximate solutions of the partial differential equation for Case 4 equation (49) with $\alpha=0.7$.

\subsection{Case 4}

The fractional diffusion equation with time dependent integral term and non homogenous term, $\sigma=1,0<\alpha \leq 1$ then equation (2) take the form

$$
\begin{aligned}
\frac{\partial^{\alpha} u(x, t)}{\partial t^{\alpha}}= & \frac{\partial^{2} u(x, t)}{\partial x^{2}}+\int_{0}^{t} u(x, s) d s+x^{2} \frac{\partial^{\alpha}}{\partial t^{\alpha}} e^{-t}-2 e^{-t}- \\
x^{2}\left(1-e^{-t}\right) & \text { (49) }
\end{aligned}
$$

Use equation (30) with equations (45), (26) and substitute them in equation (49) to get

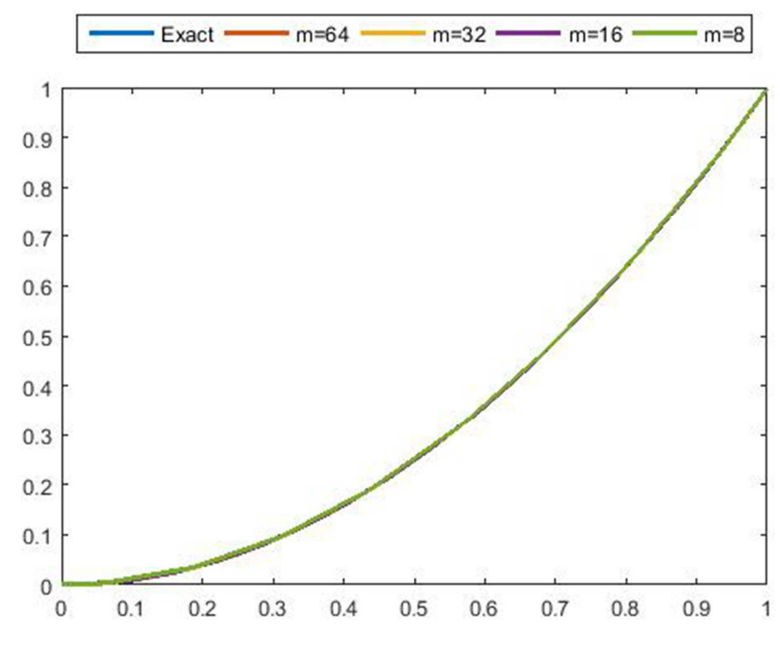

(9 a): Numerical Solutions

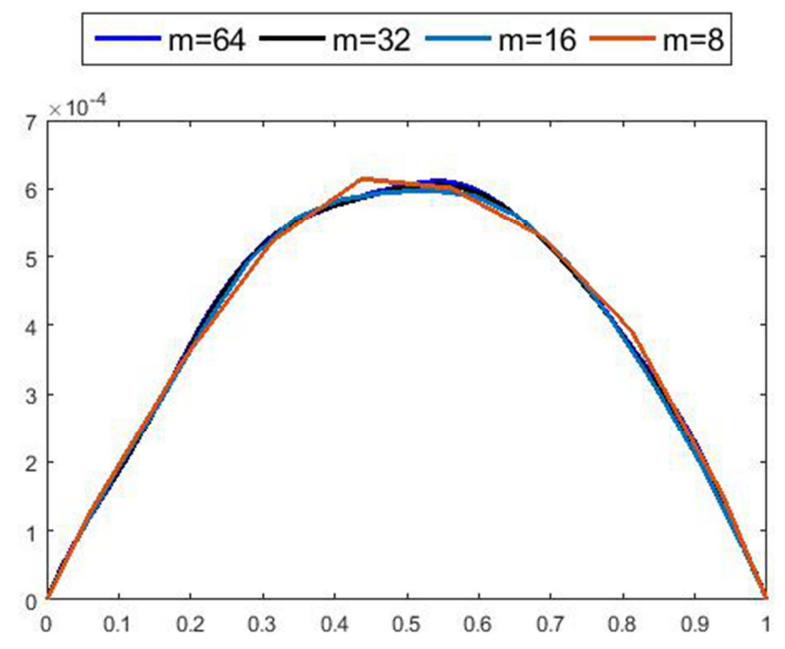

( 9 b): Maximum errors $M_{\Delta}$

Figure 9. Comparison between the exact solution and the approximate solutions of the partial differential equation for Case 4 equation (51) with $\alpha=0.9$.

$$
\begin{array}{r}
\frac{1}{\Gamma(1-\alpha)}\left(\boldsymbol{C}_{(m)}^{T} \boldsymbol{P}_{(m)}^{2} \boldsymbol{h}_{(m)}\left(x_{l}\right)-x_{l} \boldsymbol{C}_{(m)}^{T} \boldsymbol{P}_{(m)}^{2} \boldsymbol{f}\right)\left(\frac{t_{s+1}^{1-\alpha}}{1-\alpha}\right)+x_{l} \frac{\partial^{\alpha}}{\partial t^{\alpha}} e^{-t_{s+1}}-d t \boldsymbol{C}_{(m)}^{T} \boldsymbol{h}_{(m)}\left(x_{l}\right)-x_{l}^{2} \frac{\partial^{\alpha}}{\partial t^{\alpha}} e^{-t_{s+1}}+2 e^{-t_{s+1}}+ \\
x_{l}^{2}\left(1-e^{-t_{s+1}}\right)-\left(\frac{t_{s+1}^{2}}{2}-t_{s+1} t_{s}\right) \boldsymbol{C}_{(m)}^{T} \boldsymbol{P}_{(m)}^{2} \boldsymbol{h}_{(m)}\left(x_{l}\right)-t_{s+1} u\left(x_{l}, t_{s}\right)+x_{l}\left(\frac{t_{s+1}^{2}}{2}-t_{s+1} t_{s}\right) \boldsymbol{C}_{(m)}^{T} \boldsymbol{P}_{(m)} \boldsymbol{f}-t_{s+1} x_{l} e^{-t_{s+1}}- \\
x_{l}\left(1-e^{-t_{s+1}}\right)-u^{\prime \prime}\left(x_{l}, t_{s}\right)=0
\end{array}
$$

and equation (5) take the form

$$
\frac{\partial u(x, t)}{\partial t}=\frac{\partial^{1-\alpha}}{\partial t^{1-\alpha}}\left(\frac{\partial^{2} u(x, t)}{\partial x^{2}}\right)+\frac{\partial^{1-\alpha}}{\partial t^{1-\alpha}} \int_{0}^{t} u(x, s) d s-x^{2} e^{-t}+\left(x^{2}-2\right) \frac{\partial^{1-\alpha}}{\partial t^{1-\alpha}} e^{-t}
$$

Use equation (40) with equations (33), (34) and substitute them in equation (51) to get 


$$
\begin{gathered}
\boldsymbol{C}_{(m)}^{T} \boldsymbol{P}_{(m)}^{2} \boldsymbol{h}_{(m)}\left(x_{l}\right)-x_{l}\left[\boldsymbol{C}_{(m)}^{T} \boldsymbol{P}_{(m)}^{2} \boldsymbol{f}+e^{-t}\right]+\frac{t}{\alpha \Gamma(\alpha)} \boldsymbol{C}_{(m)}^{T} \boldsymbol{h}_{(m)}\left(x_{l}\right)-x_{l}^{2} e^{-t_{s+1}}+2 \frac{\partial^{1-\alpha}}{\partial t^{1-\alpha}} e^{-t_{s+1}}-\left(\frac{\Gamma(3)}{2 \Gamma(2+\alpha)} t_{s+1}^{1+\alpha}-\right. \\
\left.\frac{\Gamma(2)}{\Gamma(1+\alpha)} t_{s+1}^{\alpha} t_{s}\right) \boldsymbol{C}_{(m)}^{T} \boldsymbol{P}_{(m)}^{2} \boldsymbol{h}_{(m)}\left(x_{l}\right)-\frac{\Gamma(2)}{\Gamma(1+\alpha)} t_{s+1}^{\alpha}\left[x_{l}\left(g_{0}\left(t_{s}\right)-g_{1}\left(t_{s}\right)\right)-g_{0}\left(t_{s}\right)\right]+x_{l}\left(\frac{\Gamma(3)}{2 \Gamma(2+\alpha)} t_{s+1}^{1+\alpha}-\frac{\Gamma(2)}{\Gamma(1+\alpha)} t_{s+1}^{\alpha} t_{s}\right) \boldsymbol{C}_{(m)}^{T} \boldsymbol{P}_{(m)} \boldsymbol{f}+ \\
\left(x_{l}-x_{l}^{2}+2\right) \frac{\partial^{1-\alpha}}{\partial t^{1-\alpha}} e^{-t_{s+1}}+x_{l}^{2} e^{-t_{s+1}}-\frac{\Gamma(2)}{\Gamma(1+\alpha)} t_{s+1}^{\alpha} u\left(x_{l}, t_{s}\right)=0
\end{gathered}
$$

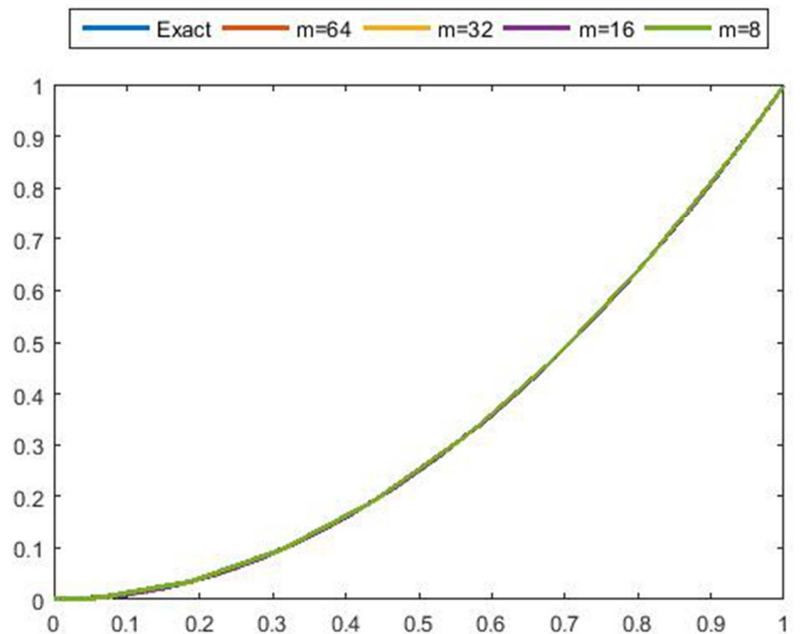

(10 a): Numerical Solutions
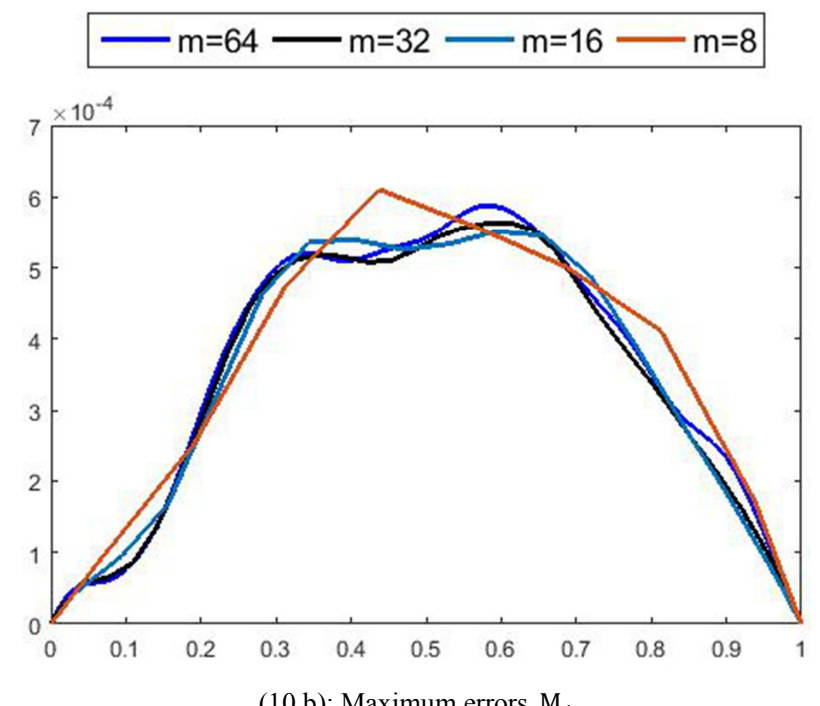

(10 b): Maximum errors $M_{\Delta}$

Figure 10. Comparison between the exact solution and the approximate solutions of the partial differential equation for Case 4 equation (51) with $\alpha=0.7$.

\section{Conclusion}

It is well known that the technique of Haar wavelet is a computer oriented method and is an acceptable method for many kinds of problems. The use of operational matrix has facilitated the complicated integrals. We have used the Haar wavelet technique to fractional time diffusion equation with non-homogeneous term and time dependent integral term. Moreover, time dependent boundary condition are considered. In the numerical calculation section the complete problem is divided into four cases ranging from the classical integer case up to the fractional order with time dependent integral term.

The use of inverse operator has improved the results significantly due to the memory effects of the fractional time derivatives which are extended to all other terms in the equation and this will be our interest with different methods in a subsequent work.

The calculated results illustrate that the wavelet techniques can be applied to many other problems. Recently, many modification in using different bases functions is used to increase the accuracy as required and this will be our objective in a subsequent work.

The small support of the considered bases functions in case of Haar wavelet demonstrates the sparsity of the resultant algebraic system.

The numerical calculations illustrated the reliability of the wavelet technique in solving PDE as shown in the figures from 1 to 10. Also, the use of the inverse operator has moved the memory effects appears in fractional derivatives to all other terms in the considered equation.

\section{References}

[1] K. Rektorys, The Method of Discretization in Time and Partial Differential Equations, Mathematics and its Applications, Springer Netherlands, 1982.

[2] I. K. Youssef, A. M. Shukur, Modified variation iteration method for fraction space-time partial differential heat and wave equations, International Journal 2 (2) (2013) 1000-1013.

[3] Ü. Lepik, Solving fractional integral equations by the haar wavelet method, Applied Mathematics and Computation 214 (2) (2009) 468-478.

[4] I. Podlubny, Fractional Differential Equations, Camb. Academic Press, San Diego, CA, 1999.

[5] K. Miller, B. Ross, An Introduction to the Fractional Calculus and Fractional Differential Equations, New York: Wiley-Interscience Publ., 1993.

[6] I. K. Youssef, R. A. Ibrahim, Boundary Value Problems, Fredholm Integral equations, SOR and KSOR Methods, Life Science Journal 10 (2) (2013) 304-312.

[7] I. K. Youssef, A. M. Shukur, Precondition for discretized fractional boundary value problem, Pure and Applied Mathematics Journal 3 (1) (2014) 1-6.

[8] I. K. Youssef, A. M. Shukur, The line method combined with spectral chebyshev for space-time fractional diffusion equation, Applied and Computational Mathematics 3 (6) (2014) 330- 336.

[9] G. D. Smith, Numerical Solution of Partial Differential Equations Finite Difference Methods, Oxford University Press, 1978.

[10] C. K. Chui, An introduction to wavelets, Vol. 1, Academic press, 2014. 
[11] Ü. Lepik, Buckling of elastic beams by the haar wavelet method, Estonian Journal of Engineering 17 (3) (2011) 271-284.

[12] C. F. Chen, C. H. Hsiao, Haar wavelet method for solving lumped and distributed-parameter systems, IEE Proc., Control Theory Appl. 144 (1) (1997) 87-94. doi: 10.1049/ip-cta: 19970702.

[13] C. F. Chen, C. H. Hsiao, Wavelet approach to optimising dynamic systems, IEE Proc., Control Theory Appl. 146 (2) (1999) 213-219. doi: 10.1049/ip-cta:19990516.

[14] Ü. Lepik, Application of the haar wavelet transform to solving integral and differential equations, Proc. Estonian Acad. Sci. Phys. Math. 56 (1) (2007) 28-46.
[15] Ü. Lepik, Numerical solution of differential equations using haar wavelets, Mathematics and computers in simulation 68 (2) (2005) 127-143.

[16] Ü. Lepik, Numerical solution of evolution equations by the haar wavelet method, Applied Mathematics and Computation 185 (1) (2007) 695-704.

[17] C. H. Hsiao, Haar wavelet direct method for solving variational problems, Mathematics and Computers in Simulation 64 (5) (2004) 569-585.

[18] C. H. Hsiao, W. J. Wang, Haar wavelet approach to nonlinear stiff systems, Mathematics and computers in simulation 57 (6) (2001) 347-353. 